\title{
Evaluation of Gamma Methods Using 2-Dimensional Ionization Array for Different Intensity Modulated Radiotherapy Cases
}

\author{
Ahmed mousa ${ }^{1}$, Rizk A. RiZK ${ }^{2}$ and Ehab M. Attalla ${ }^{3}$ \\ ${ }^{1}$ Nasser institute, helwan University, Department of physics, ${ }^{2}$ Faculty of Science, Egypt, ${ }^{3}$ National \\ cancer institute
}

Purpose: To evaluates the different gamma methods in different intensity modulated radiotherapy cases focusing on the reasons of difference in these methods.

Materials and methods: This study has performed specific intensity modulated radiotherapy quality assurance (IMRT QA) for head and neck patients (nasopharynx, thyroid, maxilla), prostate patients. IMRT plans with the same fluence map as in the treatment plan was generated on computed tomography (C.T) image of 2-dimensional phantom array, planar dose distributions perpendicular to the central axis were measured by using 2 dimensional ionization chamber array (2D array). The dose difference (DD) and distance to agreement (DTA) has been developed as gamma criteria, the gamma criteria that used in this study was $3 \mathrm{~mm}$ (DTA), $3 \%$ (DD).

Results: The mean values and standard deviations, variance of gamma results between calculated and measured map for global gamma methods were $93.5 \%, 2.7 \%, 10.7 \%$ for head and neck patients, $96.5 \%, 2 \%, 4.4 \%$ for prostate patients, respectively, mean values and standard deviation, variance for local gamma methods were $61.8 \%, 6.3 \%, 42.3 \%$ for head and neck, $57 \%, 12 \%, 141 \%$ for prostate respectively. Where acceptance value of mean gamma results are $85 \%-100 \%$.

Conclusion: These results were comparable to other studies. All the previous studies take into account only global gamma methods, the result of comparison depends on reference points of gamma index . comparison with global reference point pretends better gamma results than comparison with local reference point but is less sensitive in the low dose area, Gamma passing rate was influenced by volume of PTV, no. of segments, the range of variation in dose gradient, gamma criteria.

Key words: QA, 2D array, gamma criteria DTA, DD

Corresponding Author: Ahmed mousa_ E-mail: Mosa_science2010@yahoo.com

\section{INTRODUCTION}

Three dimensional CRT is a dramatic change from traditional practice in that it utilizes tumor and critical structures delineated on multiple transverse CT images, radiation fields designed in beam's eye view and Volumetric dose calculation and evaluation tools. IMRT is a further extension of 3D-CRT in that non-uniform fluences generated by the superposition of multiple MLC segments or dynamic MLC movements are used to improve dose conformity and OAR sparing. The modulated fluences are obtained from inverse planning and computer optimization with the guidance of clinical goals and objectives ${ }^{1}$. IMRT does allow for highly conformal dose distribution with sharp dose gradient for complex target volumes with concave surfaces. This is accomplished by the complex motion of MLC leaves equipped on a medical linear accelerator (LINAC). This advantage has been utilized to escalate dose to the tumor. As a consequence, each IMRT field has many small, irregular and asymmetric subfields defined by MLC leaves and are characterized by high dose gradient ${ }^{2}$.

Guidelines by TG 119 are available on how to verify IMRT plans. Before any IMRT treatment begins a medical physicist should verify the actual dose being delivered to the patient. This is achieved by using a phantom with a calibrated dosimetry system or films. Chambers. It is important to know that this patient specific QA is to check on the dose calculation and delivery system ${ }^{3,4}$. In general, IMRT is a complex technique including patient simulation, treatment planning, leaf sequencing, plan transfer, patient positioning, online verification and treatment deliverys. Typical patient-specific IMRT QA involves the measurement of a point dose and 2D dose distributions in a homogeneous phantom and compared with the treatment planning system (TPS) calculation. The whole delivery sequence of an IMRT treatment is transferred onto the CT image of the 
Kasr-El-Aini Journal Of Clinical Oncology And Nuclear Medicine

Vol. 9| No. 1-2 $2013 \quad$ Evaluation of Gamma Methods Using 2-Dimensional Ionization Array

phantom and calculated with TPS. The measured and calculated planar dose distributions are compared and analyzed with dose difference, distance to agreement or gamma index, etc ${ }^{6,7}$. The percentage of points, area or volume passing a pre-selected criterion is used to indicate the quality of the whole planning and delivery procedure.

Ion chamber combined with film is the early popular choice. An ion chamber could be placed in a high dose and low dose gradient region for absolute point dose measurement. Film can be irradiated to measure a relative dose distribution. An absolute planar dose map can be obtained by combining ion chamber and film measurement ${ }^{8}$. Accuracy of the measurement depends on the selection of the measuring point. This is a time consuming process. Film is gradually replaced by online $2 \mathrm{D}$ detectors such as diode arrays and ion chamber arrays. Absolute planar dose distribution could be obtained during a single delivery which makes measurement more accurate and efficient.

The gamma method, as presented by Low et al. is designed for the comparison of two dose distributions: one is defined to be the reference information (Dr (r)) (measured) and the other is queried for evaluation $(\mathrm{Dc}(\mathrm{r})$ ) calculated) based on gamma criteria DD (dose difference) and DTA (distance to agreement) between measured and calculated points dose.

A recent survey shows the majority responding clinical institutions use $3 \% / 3 \mathrm{~mm}$ criterion in their practice. At the same time, stricter criterion such as $2 \% / 2 \mathrm{~mm}$ and looser criterion such as $5 \% / 5 \mathrm{~mm}$ are both used at different institutions. With the same comparison criterion (for example, $3 \% / 3 \mathrm{~mm}$ ), there have been very different achievable agreements (or action levels) reported in literature ${ }^{8}$.

\section{AIM OF THE WORK}

The evaluation of gamma different methods in different intensity modulated radiotherapy cases focusing on the reasons of difference in these methods.

\section{MATERIALS AND METHODS}

2D Array seven 29 model (PTW-Freiburg Germany) the most tool used in this study. 2D detector array with 729 vented ionization chambers that operate at chamber voltage of $400 \mathrm{v}$, dose rate of $500 \mathrm{mGy} / \mathrm{min}$ arranged in a $27 \times 27$ matrix. The ionization chamber is plane-parallel ionization chamber of $5 \mathrm{mmx} 5 \mathrm{mmx} 5 \mathrm{~mm}$ in size of wall material made from graphite, center to center equally spacing $10 \mathrm{~mm}$, the ionization chamber is at $5 \mathrm{~mm}$ from $2 \mathrm{D}$ array surface, and ref. Point of ions chambers is $5 \mathrm{~mm}$ behind 2D array surface. Fig . 1 shows the measuring system components of $2 \mathrm{D}$ array.

xio treatment planning system (TPS) (Elekta CMS, Maryland Heights, MO) version 4.6.2 with step and shoot method in treatment planning and inverse planning software. The leaf sequencer used to convert an optimized fluence into a deliverable sequence of MLC segments.

Siemens Oncor impression linear accelerator (linac) this is a multi-energy machine (6 and $10 \mathrm{MV}$ operating up to $500 \mathrm{MU} / \mathrm{min}$ and 6 electron energies). The MLC delivery system replaces the lower movable jaws inside the linear accelerator head. The OPTIFOCUS MLC for the ONCOR linear accelerators has 41 pairs of inner leaves with a $1.0 \mathrm{~cm}$ width that projected at isocenter and two pairs of outer leaves with a $0.5 \mathrm{~cm}$ width.

2D phantom scanning; The phantom of water equivalent polystyrene was scanned via computed tomography machine (C.T) then in exactly the same way as it used for verification process, to achieve an adequate resolution during dose calculation verification, it was essential to scanned phantom with sufficiently small slice thickness, phantom scanned with $3 \mathrm{~mm}$ slice thickness then sent phantom slices to xio treatment planning system.

Treatment planning; The clinical IMRT treatment plans were designed using xio treatment planning systems. 25 patients of different sites ${ }^{14}$ nasopharynx, 3 maxilla and 4 thyroid and 4 prostate were used in this study, each case was planned by energy $6 \mathrm{MV}$. Table number (1) summary for IMRT patients including diagnosis for these patients and patient no. for each diagnosis. Table number (2) PTV was included dose prescription, total MU and no. of segments for the patients that included in table.1 (see appendix). No. of fields, beam arrangement of IMRT treatment plans were fixed for each sites separately. Fig number (2) screen shot from xio-TPS including dose distribution for PTV prescription dose 68 Gy of nasopharynx case, Fig .3 show screen shot for PTV prescription dose 60 Gy of nasopharynx case with isodose lines display as colors on transverse cut .

The phantom then transferred to XIO treatment planning system via network, Each field of imrt treatment plan is transferred separately to verification phantom, all treatment parameters are the same as for real patient plan except gantry angle which is set to zero in all beams, the field isocenter is positioned at center of ion chambers of array. Fig. 6 show screen- shot from xio -TPS including IMRT QA plan QA in coronal view.

\section{Solid phantom setup on linear accelerator:}

The measurement that was performed in this study were carried out with 2D array sandwiched between 


Vol. 9|No. 1-2 $2013 \quad$ Ahmed mousa et al.

2blocks of polystyrene plates $5 \mathrm{~cm}$ back scatter below $2 \mathrm{D}$ array, $5 \mathrm{~cm}$ build up above $2 \mathrm{D}$ array, this is the same arrangement that was designed on xio treatment planning system. The source to surface distance (SSD) was adjusted where the reference point of each chamber was $0.5 \mathrm{~cm}$ below 2D array surface then the reference point of measurement was $100 \mathrm{~cm}$ from the radiation source. in the phantom set up of measurement the gantry and collimator are set to 0 , The $2 \mathrm{~d}$ array is located on the top of package of $5 \mathrm{~cm}$ polystyrene material, adjusted in away that the isocenter is positioned at effective point of central ion. chamber then another stack of $5 \mathrm{~cm}$ polystyrene material is added above array, finally SSD of this arrangement should be 94.5 , Fig .7 show 2Darray phantom set up on ONCOR impression linear accelerator. The set up was according to C.T scan and taken proper warm up procedure i.e 15 minutes of electronic warm up, this warm up to check $2 \mathrm{D}$ array response, after warm up PTW veri-software was null to ensure a proper back ground was performed to minimize electronic noise. The output of linear accelerator was measured by farmer ioniazation chamber before IMRT -QA, Each measurement 2D array must be corrected for different air pressure and temperature.

\section{Gamma evaluation for measured and calculated plans:}

To evaluate the difference between measured, calculated matrix and the matrix dose plane at chamber plane was exported to imrt matrix software to evaluate the difference between calculated matrix that come from treatment planning system (xio), the measured matrix that come from linear accelerator through verisoft programme. While RTP file is exported to QC data base of lantis record and verification system. The data can be evaluated immediately by VeriSoft software after the field has been radiated. Various evaluation algorithms are available such as the gamma index algorithm. Recording of measurement values is completely integrated in VeriSoft.

\section{RESULTS}

Patient specific IMRT QA measurement for head and neck cancer (nasopharynx, thyroid, maxilla), prostate cancer have performed in this study using different methods of gamma evaluation, The difference between gamma evaluations methods have been tested by comparing global method with local method of gamma evaluation that measured using 2D array as dosimetric tool. The average difference between measured and calculated dose map in case of global method of gamma evaluation (mean) was $93.5 \%$ in nasopharynx patients, $95 \%$ in maxilla patients, $92.5 \%$ in thyroid patients, $96.5 \%$ in prostate patients \& in case of local method of gamma evaluation was $69 \%$ in nasopharynx, $49 \%$ in maxilla, $67.5 \%$ in thyroid, $57 \%$ in prostate, statistical analysis for all mean was shown in Fig. 8, Fig. 9, respectively. Fig.8. Mean of gamma evaluation results using global method of evaluation in different patient.

From gamma results, it was found that the S.D in these results using global method of gamma evaluation was small with maxilla patients then increase gradually to reach moderate value with prostate, nasopharynx patients then increase sharply to reach max. Value with thyroid patients while the S.D in gamma results using local method of gamma evaluation was small with thyroid patients then increase gradually to reach moderate value with maxilla, nasopharynx patients then increase sharply to reach max. Value with prostate cases, the statistical analysis that included S.D in gamma result for each patients was shown in Fig 10, Fig 11, respectively.

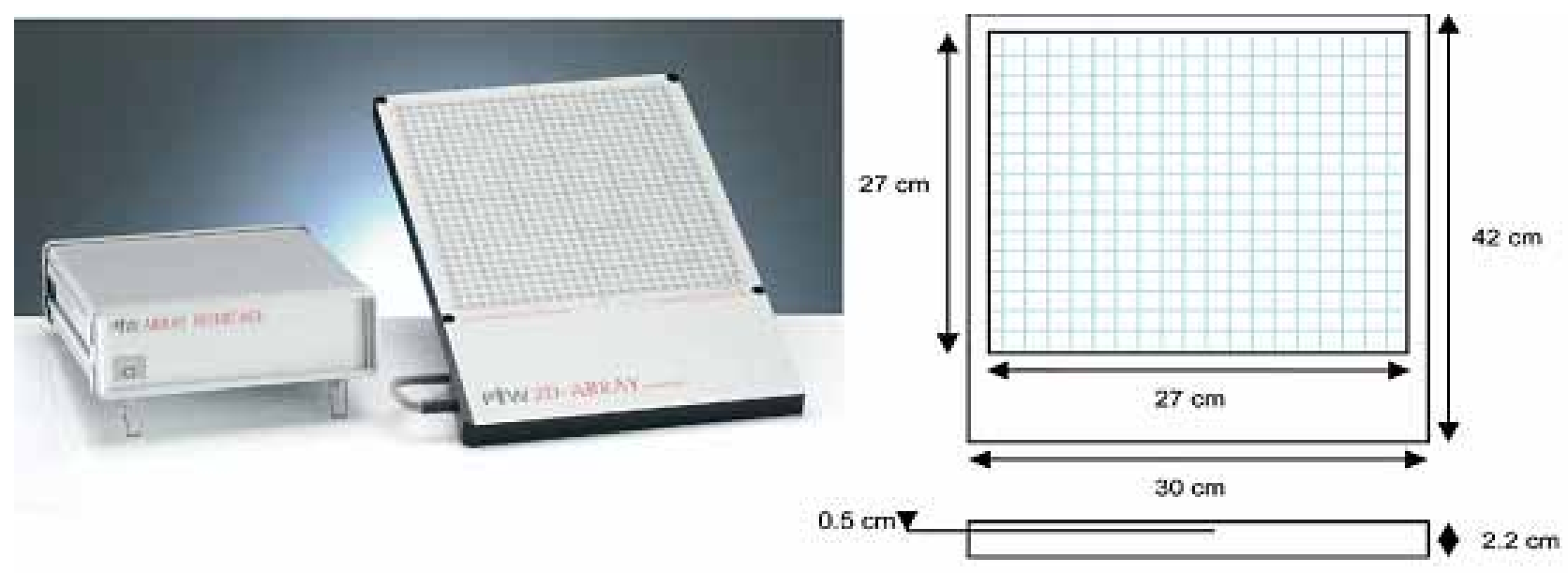

Fig.1. The major components of 2D array. 


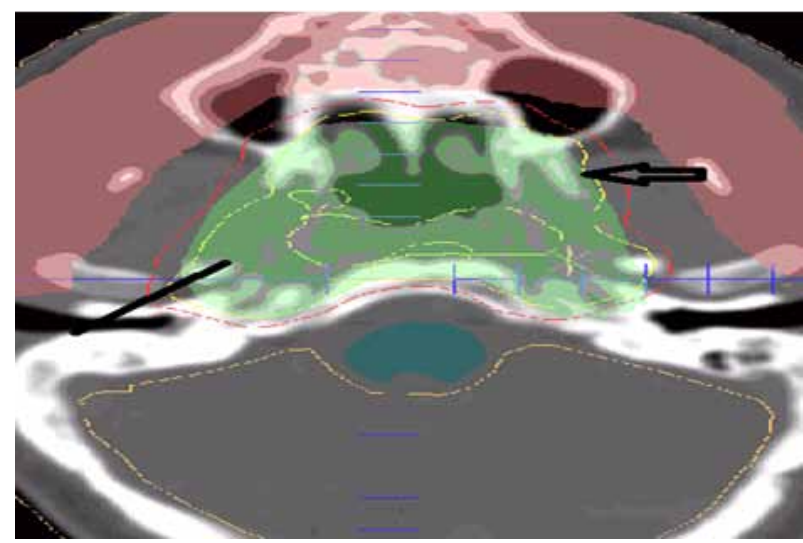

Fig. 2. the screen -shot from xio -TPS show PTV dose prescription 68 Gy of naspharynx dose Distribution with line showing PTV prescription dose 68 Gy, arrow showing 95\% isodose line.

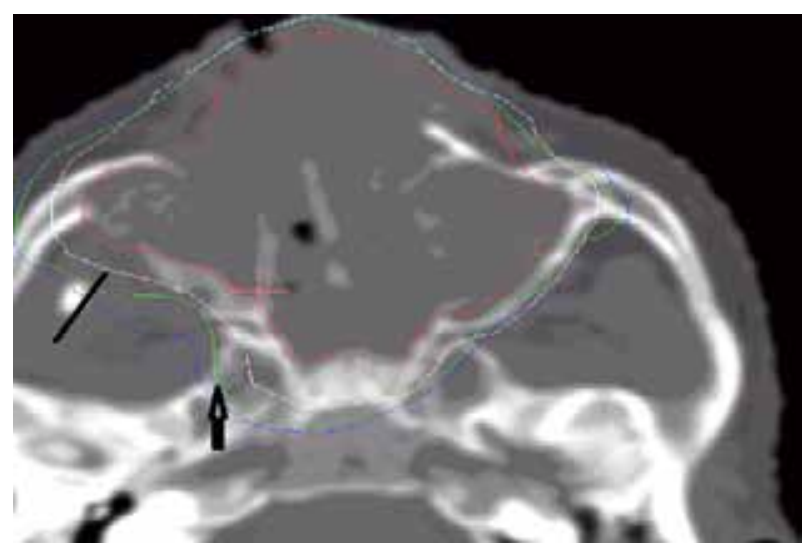

Fig. 3. Screen -shot from xio-TPS show PTV dose prescription 60 Gy of maxilla dose distribution with line showing PTV prescription dose $60 \mathrm{~Gy}$, arrow showing $95 \%$ isodose line.

Fig. 4. screen shot from xio-TPS including dose distribution for PTV dose prescription 60 Gy of thyroid case, Fig .5 screen shot for PTV dose prescription 78 Gy of prostate case with isodose lines display as colors on transverse cut.

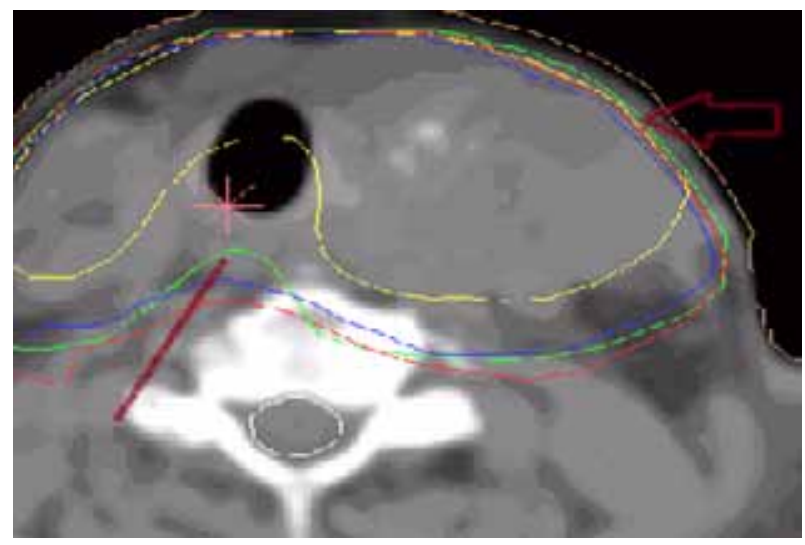

Fig. 4. Screen- shot from xio-TPS show PTV dose prescription 60 Gy of thyroid dose distribution With line showing PTV dose prescription 60 Gy Arrow showing 95\% isodose line.

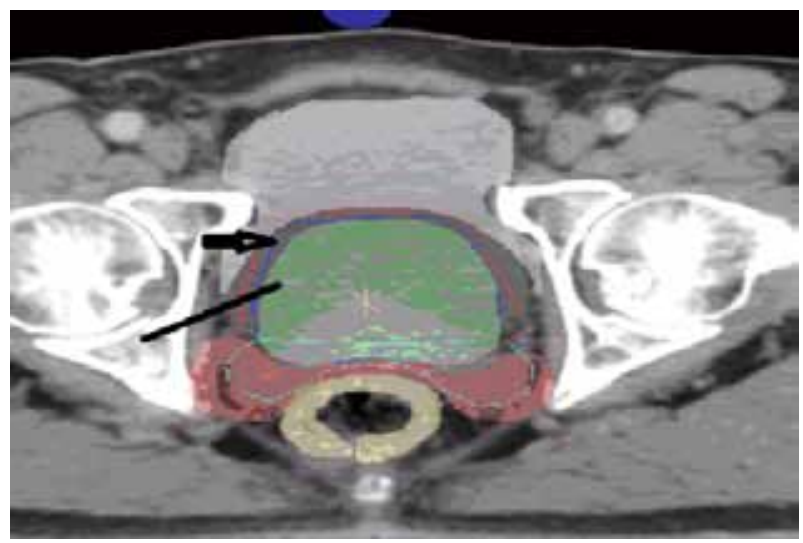

Fig. 5. Screen- shot from xio -TPS show PTV dose perscription 78Gy of prostate dose distribution With line showing PTV dose perscription $78 \mathrm{~Gy}$, arrow showing $95 \%$ isodose line.

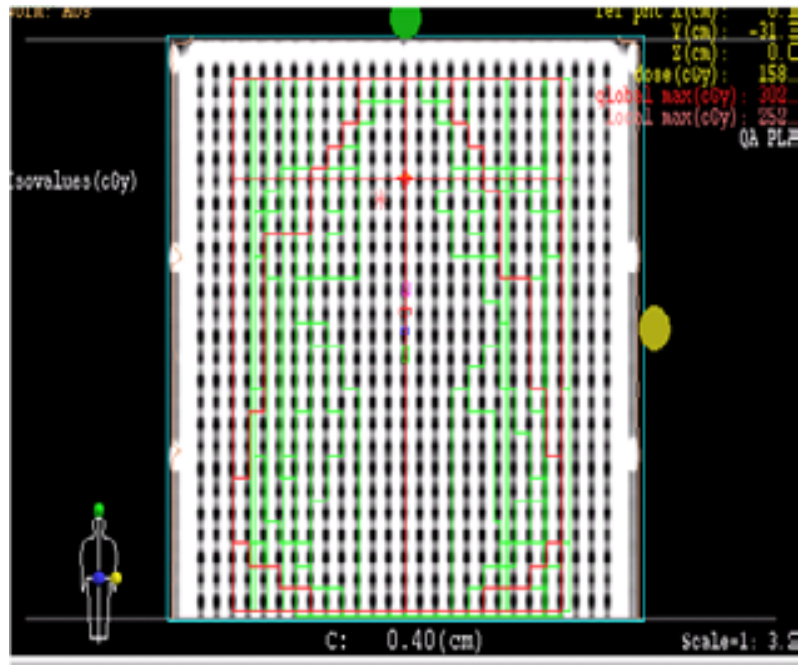

Fig. 6. screen- shot from xio -TPS show IMRT QA plan in coronal view, all gantry angle, collimator, couch were put zero angle on phantom.

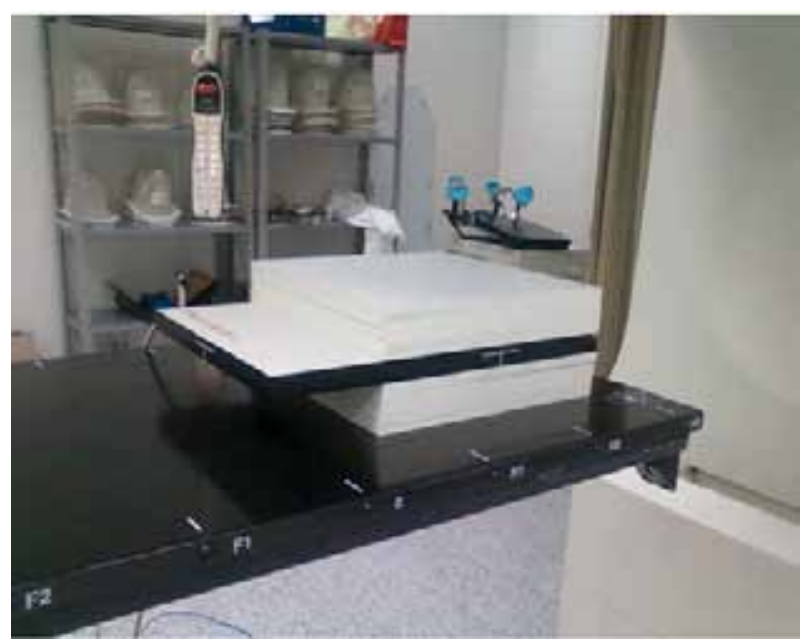

Fig. 7. 2D array phantom set up on ONCOR impression linear accelerator. 


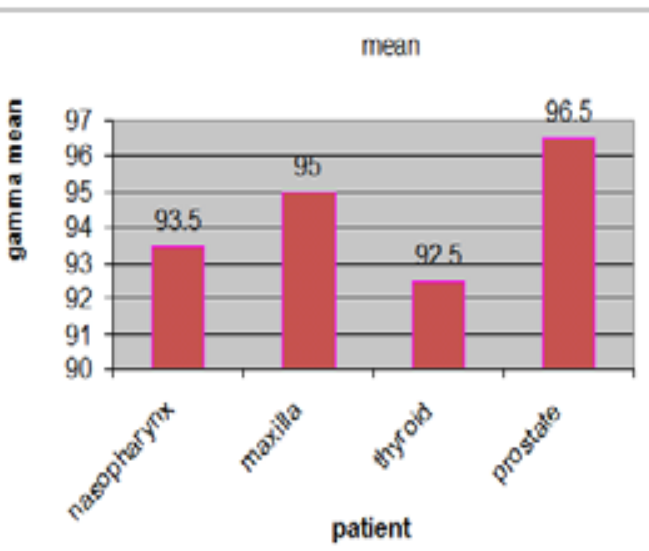

Fig. 8. Mean of gamma evaluation results using global method of evaluation in different patient.

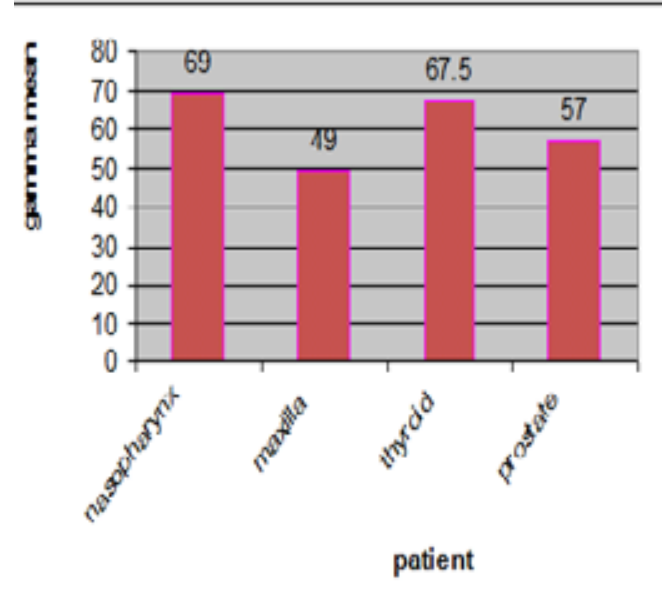

Fig. 9. Mean of gamma evaluation results using local method of evaluation in different patients.

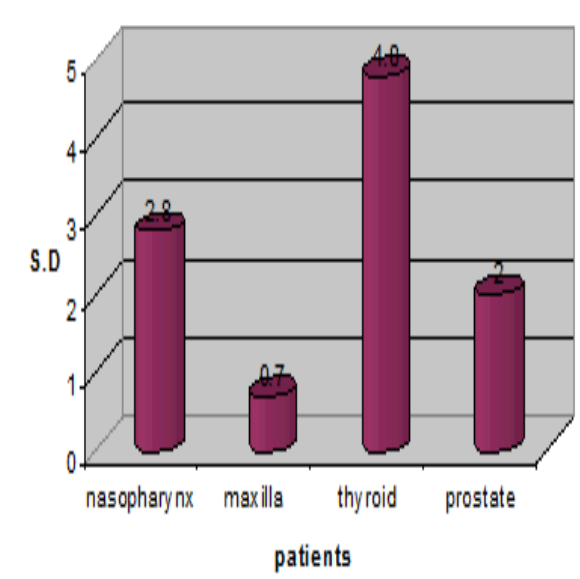

Fig. 10. Standard deviation in gamma evaluation results using global method of evaluation in different patients.

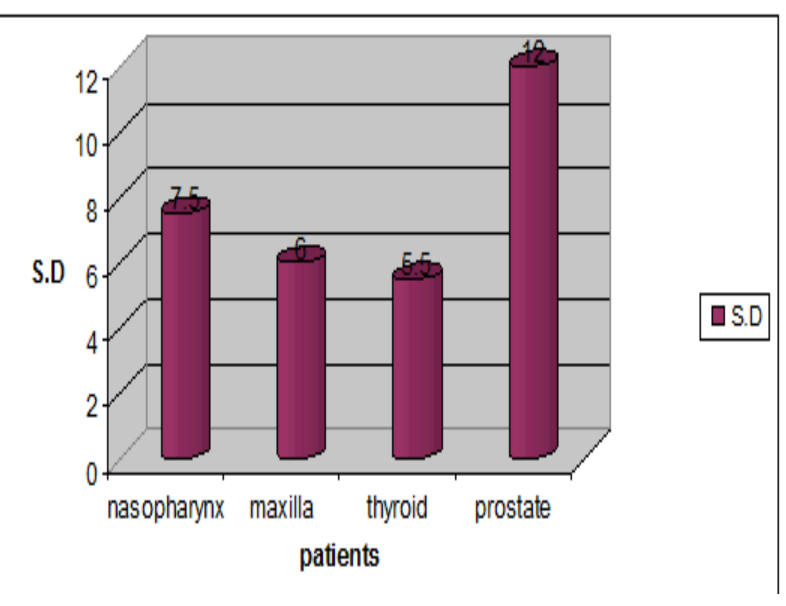

Fig. 11. Standard deviation in gamma evaluation results using local method of evaluation in different patients.

Table 1: Summary for IMRT patients including diagnosis, no. of patients

\begin{tabular}{lc}
\hline Diagnosis & No. of cases \\
\hline nasopharynx & $14(1-14)$ \\
\hline maxilla & $3(15-17)$ \\
\hline thyroid & $4(18-21)$ \\
\hline prostate & $4(22-25)$ \\
\hline
\end{tabular}

\section{Appendix}

Table 2. Showing diagnosis, no. of segments and total MU per fraction for each patient.

\begin{tabular}{|c|c|c|c|}
\hline & Diagnosis & No. of Segments & $\begin{array}{c}\text { Total } M U / \\
\text { fraction }\end{array}$ \\
\hline Case1 & nasopharynx & 76 & 839 \\
\hline Case2 & nasopharynx & 129 & 844 \\
\hline Case 3 & nasopharynx & 71 & 611 \\
\hline Case4 & nasopharynx & 90 & 1000 \\
\hline Case 5 & nasopharynx & 120 & 778 \\
\hline Case6 & nasopharynx & 93 & 826 \\
\hline Case7 & nasopharynx & 130 & 1500 \\
\hline Case8 & nasopharynx & 106 & 1120 \\
\hline Case9 & nasopharynx & 150 & 900 \\
\hline Case10 & nasopharynx & 128 & 723 \\
\hline Case11 & nasopharynx & 169 & 123 \\
\hline Case12 & nasopharynx & 100 & 180 \\
\hline Case13 & nasopharynx & 146 & 200 \\
\hline Case14 & nasopharynx & 146 & 1500 \\
\hline Case15 & maxilla & 102 & 1500 \\
\hline Case 16 & Maxilla & 73 & 1500 \\
\hline Case17 & Maxilla & 69 & 1300 \\
\hline Case18 & Thyroid & 121 & 1200 \\
\hline Case19 & Thyroid & 119 & 780 \\
\hline Case20 & Thyroid & 53 & 1100 \\
\hline Case21 & Thyroid & 76 & 1600 \\
\hline Case22 & prostate & 76 & 527 \\
\hline Case23 & prostate & 35 & 606 \\
\hline Case24 & prostate & 64 & 626 \\
\hline Case25 & prostate & 62 & 690 \\
\hline
\end{tabular}


Kasr-El-Aini Journal Of Clinical Oncology And Nuclear Medicine

Vol. 9 | No. 1-2 $2013 \quad$ Evaluation of Gamma Methods Using 2-Dimensional Ionization Array

Table 3: summary for S.D, mean, variance for gamma evaluation result for global method.

\begin{tabular}{lcc}
\hline & SD & Mean \\
\hline Nasopharynx & 2.8 & 93.5 \\
\hline Maxilla & 0.7 & 95 \\
\hline Thyroid & 4.8 & 92.5 \\
\hline Prostate & 2 & 96.5 \\
\hline
\end{tabular}

Table 4: summary for S.D, mean, variance for gamma evaluation results for local method.

\begin{tabular}{lcc}
\hline & SD & mean \\
\hline Nasopharynx & 7.5 & 69 \\
\hline Maxilla & 6 & 49 \\
\hline Thyroid & 5.5 & 67.5 \\
\hline Prostate & 12 & 57 \\
\hline
\end{tabular}

\section{DISCUSSION AND CONCLUSION}

\section{From these results it was found that:}

\section{1) In global gamma method}

Gamma passing rate in one of nasopharynx patient was lager $(96.4 \%)$ than another nasopharynx patient $(91.8 \%)$ where no. of segments was smaller in first nasopharynx patient (106 segments) than the second nasopharynx patient (130 segments), The gamma passing rate in one of maxilla patient was larger $(95.7 \%)$ than another maxilla patient $(95.1 \%)$ where no. of segments was smaller in first maxilla patient ( 73 segments) than another maxilla patient (102 segments), The gamma passing rate in one of prostate patient was larger $(98.8 \%)$ than another prostate patient $(96.7 \%)$ where no. of segments was smaller in first maxilla patient (73segments) than another maxilla patient (102 segments). These results were related to two main reasons.

1 -Volume of PTV in nasopharynx, thyroid patients is larger than volume of PTV in prostate, maxilla patients hence no of segments increase in nasopharynx, thyroid patients than maxilla, prostate patients to fitted these increase in PTV volume.

2-When no of segments increase, the uncertainty in MLC performance increase hence uncertainty in leaf positioning increase, error in point dose of gamma matrix increase, agreement between measured and calculated matrix decrease, the gamma passing rate decrease. 3-high dose gradient near the point of measurement in high modulated head and neck plans, with this high dose gradient, set up error can attribute to large deviation of gamma results, in prostate the gamma passing rate was superior to $96.5 \%$, this result was mainly due to the fact that the modulation of beam for prostate generate uniform dose distribution that was correlated to good passing rate.

From these results also it was found that larger variation in gamma passing rate results in nasopharynx patients $(96.4 \%, 91.85 \%)$, thyroid patients $(92.7 \%, 95.2 \%)$, small variation in gamma passing rate results in maxilla patients $(95.7 \%, 95.1 \%)$, prostate patients $(96.7 \%, 98.8 \%)$ than in maxilla patients, these variation in results were correlated to that presence of large no. of organ at risk (OAR) at near distance from PTV in nasopharynx patients, thyroid patients as parotid, cochlea and mucosa and brain stem in nasopharynx patients, larynx, esophagus in thyroid patients, presence of few no. of at near distance from PTV in maxilla, prostate patients as optic chiasm, optic nerve, brain stem in maxilla patients, as rectum, bladder in prostate patients, when organ at risk near PTV increase, this require large variation in dose gradient between PTV, organ at risk to achieve that max dose reach to PTV, less dose reach to organ at risk, this require variation in intensity map hence variation in no of segments, this lead to variation in gamma passing rate results.

\section{2) In local gamma method}

Gamma passing rate in one of nasopharynx patients was $73 \%$, in another nasopharynx patients was $75.8 \%$, in one of thyroid patients was $64.6 \%$, in another thyroid patients was $64.3 \%$, Gamma passing rate in one of maxilla patients was $47.5 \%$, in another thyroid case patients $41.3 \%$, Gamma passing rate in one of prostate patients was $57.7 \%$, in another thyroid patients was $78.4 \%$.

\section{These results were related to main reasons.}

1-Volume of PTV in prostate, maxilla patients is smaller than volume of PTV in nasopharynx, thyroid patients hence no. of evaluated points in gamma matrix are smaller in prostate, maxilla than nasopharynx, thyroid patients that have large no. of evaluated point. In local method of evaluation each value of measured matrix was compared with each value of calculated matrix but without normalization relative to max. Value, hence the presence of large no. of evaluated points in measured, calculated matrix increase the ratio of passing points than failing points the same as in nasopharynx, thyroid patients, on the contrast in prostate, maxilla cases the presence of small no. of evaluated points in measured, calculated matrix decrease the ratio of passing points than failing point. 
2- The presence of large variation in gamma results in one of prostate patients from another patient due to that; decrease volume of PTV, hence decrease no. of evaluated points result in that any small change in volume of PTV or OAR that surround the PTV from prostate case to another can cause large variation in no. of evaluated points, hence the ratio of passing and failing points, hence can affect on gamma results. The gamma passing rate results are better in case of gamma evaluation with global method of evaluation than in case of gamma evaluation with local method of evaluation, the no of failing points in gamma matrix is larger in case of relative method of evaluation than in case of absolute method of evaluation.

This result was correlated that; in global method of evaluation each value of measured matrix was compared with each value of calculated matrix after normalization relative to max. value in each matrix, this normalization decreases the possibility of miss matching between measured and calculated values hence gamma passing rate results increase. In local method of evaluation each value of measured matrix was compared with each value of calculated matrix but without normalization relative to max. value, absence of normalization increase the miss matching between measured and calculated values hence the gamma passing rate results decrease.

\section{3) In prescribed (selected) dose method:}

Gamma results are the same as global gamma method, this related to that max. Value that inserted in prescribed dose gamma method is the same max. value that used in global gamma method as ref. value, rest of points in gamma matrix is relative to this ref. point so prescribed gamma method give the same results as absolute gamma method.

Jin Beom Chung, Jae Sung Whan Ha and Sung-Joon Ye 2011 have been performed patient specific IMRT QA measurement for head and neck cancer, brain tumor and prostate cancer. Treatment plans were optimized with eclipse treatment planning system using a diode array as dosimetric tool. These measurement have been performed with Varian linac equipped with 120 MLC for the treatment delivery using sliding window IMRT technique and gamma criteria of $3 \% / 3 \mathrm{~mm}$, using global method as gamma evaluation method. But this study has been investigated global and local, prescribed (selected) dose gamma evaluation methods using gamma criteria $3 \% / 3 \mathrm{~mm}$, 2D ionization chamber array as dosimetric tool. Treatment plans were optimized with xio treatment planning system, siemens oncor linac were used for treatment delivery using step and shoot IMRT technique.

-Daniela Wagner, Hilke Vorwerk 2011 have been performed patient specific IMRT QA measurement for head and neck cancer and prostate cancer using 2D ionization chamber array as dosimeric tool, patient rapid arc treatment plans using gamma criteria $3 \% / 3 \mathrm{~mm}$ and global method gamma evaluation These measurements took place with varian linac, Treatment plans were optimized with eclipse treatment planning system. But this study has been investigated global and local, prescribed (selected) dose gamma evaluation methods. Treatment plans were optimized with xio treatment planning system, siemens oncor linac were used for treatment delivery using step and shoot IMRT technique using the same gamma criteria, 2D ionization chamer as dosimetric tool.

\section{CONCLUSION}

Gamma passing rate results were influenced by volume of PTV, number of segments, the range of variation in dose gradient where mean of gamma passing rate results were $93.5 \%$ in nasopharynx case with large PTV while the mean of gamma passing rate results were $96.5 \%$ in prostate case with small PTV and standard deviation in gamma results were large in nasopharynx case $2.8 \%$ while standard deviation in gamma results were small in prostate case $2 \%$ where variation in dose gradient was large in nasopharynx case than prostate case. The ability of $2 \mathrm{D}$ array to simultaneously perform global and local methods can simplify reduce IMRT work load, high dose gradient in head and neck case was related to that the modulation factor was greater due to increased complexity required to reach constrains, this was in agreement with poor gamma passing rate results.

Prostate patients have good gamma passing rate results, this was mainly due to the fact that modulation factor was smaller, simplification required to reach constrain that generate more uniform dose distribution. PTW 2-dimensinal array soft ware verisoft offer the selection of global ref dose value, Local dose ref. value gamma evaluation, selected dose value, in local method the comparison was done between every single point on calculated, measured matrix without normalization to another ref value, dose at corresponding position of ref. matrix is compared, In global method the comparison was done after normalization for each point on matrix relative to another relative value, this value is the max. Dose of ref. Value, selected dose method is the same as global method, the result of comparison depends on reference points of gamma index, comparison with global reference point pretends better gamma results than comparison with local reference point but is less sensitive in the low dose area. Where mean value of gamma results in global method of evaluation in nasopharynx, prostate were $93.5 \%, 96.5 \%$, respectively, in case of local method of evaluation were $69 \%, 57 \%$, respectively. 


\section{REFERENCES}

1. Galvin JM, Ezzell G, Eisbrauch A, Yu C, Butler B, Xiao Y, et al. Implementing IMRT in clinical practice: A joint document of the American Society for Therapeutic Radiology and Oncology and the American Association of Physicists in Medicine. Int.J.Radiat.Oncol.Biol.Phys. 2004;58(5):1616-34.

2. Palta JR, Liu C, Li JG. Quality assurance of intensitymodulated radiation therapy. Int.J.Radiat.Oncol.Biol. Phys. 2008;71(1 Suppl):S108-12.

3. Ezzell GA, Galvin JM, Low D, Palta JR, Rosen I, Sharpe MB, et al. Guidance document on delivery, treatment planning and clinical implementation of IMRT: Report of the IMRT Subcommittee of the AAPM Radiation Therapy Committee. Med.Phys. 2003;30(8):2089-115.

4. Bernan. Transition from 2-D Radiotherapy to 3-D Conformal and Intensity Modulated Radiotherapy: IAEA Tecdoc Series No. 1588. International Atomic Energy Agency; 2008.

5. Ezzell GA, Galvin JM, Low D, Palta JR, Rosen I, Sharpe $\mathrm{MB}$, et al. Guidance document on delivery, treatment planning and clinical implementation of IMRT: Report of the IMRT Subcommittee of the AAPM Radiation Therapy Committee. Med.Phys. 2003;30(8):2089-115.

6. Low DA, Harms WB, Mutic S, Purdy JA. A technique for the quantitative evaluation of dose distributions. Med. Phys. 1998;25(5):656-61.
7. Van Dyk J, Barnett RB, Cygler JE, Shragge PC. Commissioning and quality assurance of treatment planning computers. Int.J.Radiat.Oncol.Biol.Phys. 1993;26(2):261-73.

8. Nelms BE, Simon JA. A survey on planar IMRT QA analysis. J.Appl.Clin.Med.Phys. 2007;8(3):2448.

9. Spezi E, Angelini AL, Ferri A. A multiple acquisition sequence for IMRT verification with a $2 \mathrm{D}$ ion chamber array. Med.Dosim. 2006;31(4):269-72.

10. Spezi E, Angelini AL, Romani F, Ferri A. Characterization of a $2 \mathrm{D}$ ion chamber array for the verification of radiotherapy treatments. Phys.Med.Biol. 2005;50(2005):336-3373.

11. Chung JB, Kim JS, Ha SW, Ye SJ. Statistical analysis of IMRT dosimetry quality assurance measurements for local delivery guideline. Radiat.Oncol. 2011;6:27.

12. Wagner D, Vorwerk H. Two years experience with quality assurance protocol for patient related Rapid Arc treatment plan verification using a two dimensional ionization chamber array. Wagner Vorwerk Radiat.Oncol. 2011;6:21.

13. Fenoglietto $P$, Laliberté $B$, Aillères $N$, Riou $O$, Dubois JB, Azria D. Eight years of IMRT quality assurance with ionization chambers and film dosimetry: Experience of the Montpellier Comprehensive Cancer Center. Radiat. Oncol. 2011(6):85.

14. Stasi M, Giordanengo S, Cirio R, Boriano A, Bourhaleb F, Cornelius I, et al. D-IMRT verification with a 2D pixel ionization chamber: Dosimetric and clinical results in head and neck cancer. Phys.Med.Biol. 2005;50(19):4681-99. 